\title{
Watermarked Compressive Sensing Measurements Reconstructed by the Greedy Algorithms
}

\author{
Ali A. H. Karah Bash and Sema K. Kayhan
}

\begin{abstract}
This paper proposes a CS based watermarking algorithm. In this algorithm, the watermarking image embeds into the compressive measurement vectors by the encoder. The measurement vectors are sparse in suitable basis. The resulting watermarked measurements recover by using both the OMP and the OMP-PKS reconstruction algorithms. Then the decoder uses to extract the watermarking image.

In an experimental study, results obtained by the OMP and the OMP-PKS algorithms are compared. The results show that the OMP-PKS algorithm achieves performance superior to that of the OMP reconstruction algorithm.
\end{abstract}

Index Terms-Watermarking image, CS, OMP and OMP-PKS algorithms.

\section{INTRODUCTION}

In the Shannon-Nyquist sampling approach, a signal data reconstructs if the signal is sampled at the rate at least twice higher than the maximum frequency in the signal. Storing or transmission these signals can be difficult because of large size, sometimes it is expensive and not reasonable.

The recent studies use the Compressive Sensing CS [1]-[4]. In CS, the signals can be reconstructed by using a small number of random measurements that is called sensing matrix and the signals must be sparse. The sparse image contains a few coefficients as non-zero and other coefficients are zero.

However, the natural images almost are not sparse. Therefore, some transformation methods such as DCT, DFT and DWT utilize to convert these natural images into sparse images. The CS image reconstruction bases on different methods such as the optimization and the greedy methods.

In this study, two greedy algorithms use to reconstruct the sparse image, the orthogonal matching pursuit OMP and orthogonal matching pursuit with partially known support OMP-PKS [5]-[8]. The fundamental difference between the OMP and the OMP-PKS is that the OMP-PKS has a prior knowledge support about some coefficients of the sparse image; and the other coefficients are unknown support. Moreover, the ability to recover the sparse image from a small band of information is achieved.

The digital watermarking provides data protection [9], [10]. In the digital watermarking, the embedded algorithm (encoder) inserts any information or data to the measurement matrix.

Manuscript received February 16, 2014; revised May 21, 2014

Ali A. H. Karah Bash and Sema K. Kayhan are with Gaziantep University, Turkey (e-mail: al_muhands2011@yahoo.com, skoc@gantep.edu.tr).
After sparse image reconstruction, the decoder detects a successful watermark. The paper is organized as follows: Section I gives an introduction. Section II explains the basic concepts of CS approach. Section III presents the encoder and decoder algorithms to insert and extract the watermarking image into the watermarked measurements. Section IV shows the experimental results and the conclusion presents in Section V.

\section{COMPRESSIVE SENSING}

A signal $x \in R^{N}$ is a $k$-sparse when the most $k$ elements of $x$ are non-zero. Let $f \in R^{N}$ is a $k$-sparse signal in the $\psi$ space that is a linear combination of $N, \psi$ represents an orthonormal basis and $f$ shows as:

$$
f=\psi x .
$$

Then the signal $x$ can be represented as a sparse signal by using $x=\psi^{\prime} f$ and one can sample the information as [11], [12]:

$$
y=\varnothing f
$$

where $y \in R^{M}$ is a measurement vector and $\varnothing$ is an $M \mathrm{x} N$ sensing matrix. Then the previous equation rearranges as:

$$
y=\varnothing f=\varnothing \psi x=\phi x .
$$

where $\phi \in R^{M x N}$ represents as underdetermined matrix, with $M \ll N$. The reconstruction method estimates the signal $\widehat{x}$ that is at most $k$ values are non-zero and $k<M \ll N$. If the matrix $\phi$ satisfies the Restricted Isometry Property (RIP), $\hat{x}$ can be exactly reconstructed using the OMP and OMP-PKS algorithms. Candès and Tao introduced RIP in [13] as follows:

Definition: A matrix $\phi$ satisfies RIP of order $k$ if there exists a $\delta_{k} \in(0,1)$ such that:

$$
\left(1-\delta_{k}\right)\|x\|_{2}^{2} \leq\|\phi x\|_{2}^{2} \leq\left(1+\delta_{k}\right)\|x\|_{2}^{2} .
$$

where $\delta_{k}$ is the Restricted Isometry Constant RIC. If $\phi$ satisfies the RIP of order $2 k$ with $\delta_{2 k}<\sqrt{2}-1$, the $k$ sparse vector $x$ given in equation (3) can reconstruct by using the OMP and the OMP-PKS algorithms: 


$$
\widehat{x}=\arg \min _{x}\|x\|_{1} \text { St. } y-\phi x=0 .
$$

When $\phi$ is a random Gaussian matrix, then $M=\mathrm{O}(k) \log$ $(N / k)$ measurements represent the row of the sensing matrix.

\section{WATERMARKING ALGORITHM FOR THE CS MEASUREMENTS}

The watermarking algorithm used in this study is same with the algorithm in [14], but for the reconstruction, we used OMP and OMP-PKS algorithms instead of $l_{1}$ minimization method.

The watermarking process includes three algorithms: encoder, reconstruction and decoder.

\section{A. The Encoder}

In the encoder process, a watermark $v$ embeds into the Compressive Sensed Measurements of a sparse signal $x$. The watermarking image represents as $v \in\{-\alpha,+\alpha\}^{L}, L$ is the number of bits, and the watermarked measurement vector symbolizes as $y_{v} \in R^{M}$ :

$$
y_{v}=\phi x+D v \text {. }
$$

where $D$ is a random matrix with $M \times L$, and it must be known prior to the insertion and extraction procedures. The watermarked measurements may be changed by parasitical or by channel defects and the decoder receives:

$$
y_{n}=\phi x+D v
$$

That reorders as:

$$
y_{v}=H\left[\begin{array}{l}
x \\
v
\end{array}\right] \text {, such that } H=[\phi \mid D] .
$$

\section{B. The Reconstruction Algorithms}

The OMP is a greedy algorithm that is developed version of the matching pursuit MP [15]. The OMP estimates the magnitude of the nonzero coefficients of $x$ by solving the least square error between the orthogonal projection of the recovered $x$ and measurement vector $y$.

The watermarking compressive sensing measurement $y_{v}$ includes both $k$-sparse $x$ and $L$ bit watermarking image $v$, then $y_{v}$ reconstructs by using the OMP and the OMP-PKS algorithms from the computed $y_{n}$ by using equation (7).

$$
\widehat{x}=\arg \min _{x}\|y-\phi x\|_{2} .
$$

It changes as: $\hat{Z}=\arg \min _{x}\left\|y_{v}-H Z\right\|_{2}$.

where: $\quad \hat{Z}=\left[\begin{array}{c}\tilde{x} \\ \tilde{v}\end{array}\right]$ and $Z=\left[\begin{array}{c}x \\ v\end{array}\right]$.
The OMP-PKS [16] is a greedy algorithm uses for sparse image reconstruction and it is developed from the traditional OMP algorithm. It uses the sparse image that has some components more important than the others.

The OMP-PKS successfully reconstructs $y$, which is defined in equation (3), where the $M$ dimensions of $y$ are very small, that mean the OMP-PKS has a very low measurement rate $(M / N)$.

The sparse image generates from discrete wavelet transform (DWT) [17]. Let $\mathrm{T}$ is a set includes the indexes of the approximation LL sub-bands and represents as known support part that all components of it are nonzero, $\mathrm{T}=\left\{\gamma_{1}, \gamma_{2}, \ldots, \gamma_{|T|}\right\}$.

The procedure of the OMP-PKS algorithm divides into two parts; the first part uses the partially known support, which provides a priori knowledge to compute the important sub-bands in the sparse image.

$$
\begin{gathered}
\mathrm{T}=\text { (index of known part), } \mathrm{T}=\left\{\gamma_{1}, \gamma_{2}, \ldots, \gamma_{|T|}\right\} . \\
H=\left[\varphi_{\gamma_{1}}, \varphi_{\gamma_{2}}, \varphi_{\gamma_{3}} \ldots \ldots \ldots, \varphi_{\gamma_{|T|}}\right] . \\
Z=\left(H^{?} H\right)^{-} H y_{v} . \\
a_{|T|}=\langle H Z\rangle . \\
r=y_{v}-a_{|T|} \text { or } r=y_{v}-H_{T}\left(H_{T}^{\dagger} y\right) .
\end{gathered}
$$

The second part shows the OMP algorithm.

\section{The Decoder}

The decoder utilizes for the watermarking image extraction. After reconstruction of the watermarking compressive sensing measurements, the decoder uses to extract the watermark as [18]:

$$
\begin{gathered}
\hat{Z}=\arg \min _{x}\left\|y_{v}-H Z\right\|_{2},\left[\begin{array}{c}
\tilde{x} \\
\tilde{v}
\end{array}\right]=\hat{Z} . \\
\hat{v}=\alpha^{*} \operatorname{sgn}(\tilde{v}) . \\
\widehat{x}=\arg \min _{x}\left\|\left(y_{v}-D \widehat{v}\right)-\phi x\right\|_{2} .
\end{gathered}
$$

where $\hat{x}$ is the recovered image and $\widehat{v}$ is the extracted watermark image.

\section{EXPRIMENTAL RESULtS}

In the experimental study, at first, the watermarking image embeds into the CS measurements, then the watermarked measurements reconstruct by OMP and OMP-PKS algorithms, and finally watermarking image successfully extracts. Results compare performance both OMP and OMP-PKS algorithms using $64 \times 64$ watermark image and $256 \times 256$ Lena image. The performance evaluation bases on PSNR of the reconstructed image.

Fig. 1 with Fig. 2 show Lena and watermarking images respectively. Fig. 3 and Fig. 4 show the recovery quality of Lena and watermarking images obtained by OMP and 
OMP-PKS algorithms respectively for a different measurement rate $(M / N)$. Fig. 5 represents PSNR, MSE and residual (error) values for varying number of measurement rates obtained by OMP and OMP-PKS. Fig. 6 shows the performance of OMP and OMP-PKS approaches by computing the PSNR values for different number of non-zero coefficients $k$, where $k$ varies between (5506 to 9433).

The results in Table I show that the OMP algorithm has a steady state at $M / N=0.528$ with $\mathrm{PSNR}=29.885$. While OMP-PKS algorithm has a steady state when $M / N=0.293$ with $\mathrm{PSNR}=36.854$. The higher values of $M / N$ after the steady state rate give same PSNR value. As it is seen, OMP requires higher measurement rates than the OMP-PKS algorithm to obtain reasonable PSNR values. Therefore, the OMP-PKS has a better performance than the OMP.

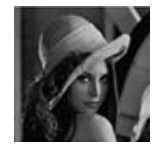

Fig. 1. Original image.
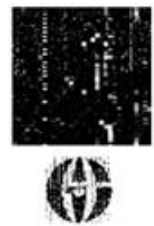

M/N rates: 0.176 PSNR values:7.708
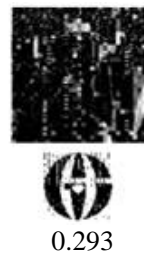

8.565

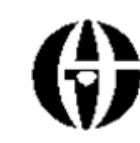

Fig. 2. Watermark image.
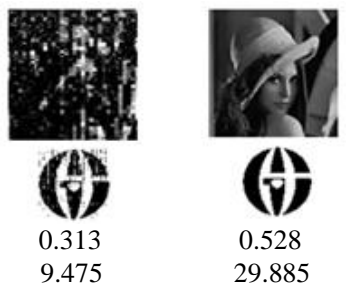

0.528 29.885

Fig. 3. Quality of recovered and watermarking images in different $\mathrm{M} / \mathrm{N}$ rates by OMP.

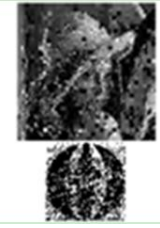

0.176

$\mathrm{M} / \mathrm{N}$ rates:

PSNR values: 10.115

Fig. 4. Quality of recovered and watermarking images in different $\mathrm{M} / \mathrm{N}$ rates by OMP-PKS.

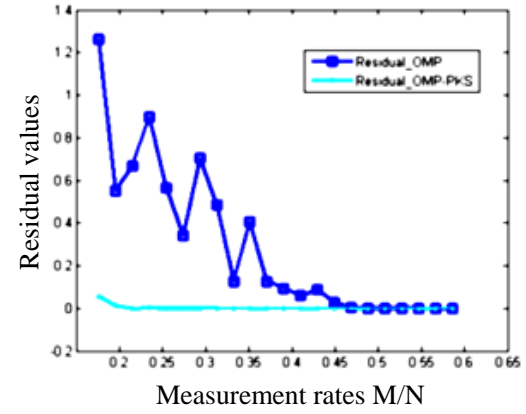

(a)

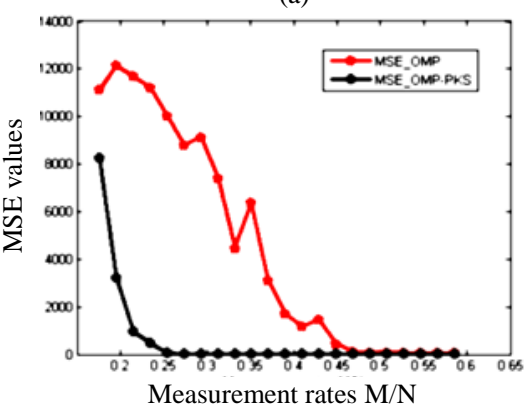

(b)

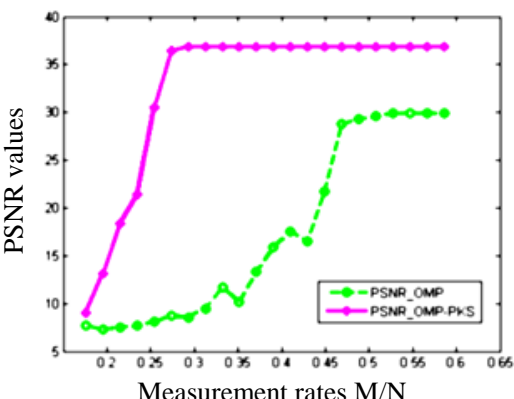

(c)

Fig. 5. (a). Residual, (b). MSE and (c). PSNR values, respectively for varying measurement rates $\mathrm{M} / \mathrm{N}$.

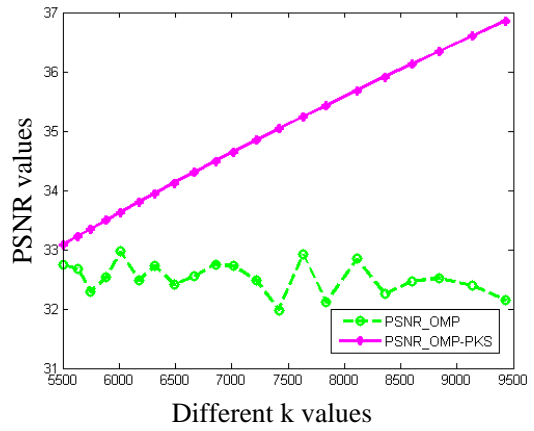

Fig. 6. Performance OMP and OMP-PKS algorithms for different $\mathrm{K}$ values.

TABLE I: PERFORMANCE OF OMP AND OMP-PKS ALGORITHMS FOR VARYING M/N VALUES

\begin{tabular}{cccc}
\hline \hline \multicolumn{2}{c}{ OMP algorithm } & \multicolumn{2}{c}{ OMP-PKS algorithm } \\
\hline $\begin{array}{c}\text { M/N } \\
\text { values }\end{array}$ & PSNR values & $\begin{array}{c}\text { M/N } \\
\text { values }\end{array}$ & PSNR values \\
\hline 0.176 & 7.708 & 0.176 & 10.115 \\
0.196 & 7.324 & 0.196 & 12.748 \\
0.215 & 7.486 & 0.215 & 17.224 \\
0.235 & 7.668 & 0.235 & 23.289 \\
0.254 & 8.154 & 0.254 & 23.670 \\
0.274 & 8.728 & 0.274 & 29.955 \\
0.293 & 8.565 & 0.293 & 36.854 \\
0.313 & 9.475 & 0.313 & 36.854 \\
0.333 & 11.677 & 0.333 & 36.854 \\
0.352 & 10.131 & 0.352 & 36.854 \\
0.372 & 13.290 & 0.372 & 36.854 \\
0.391 & 15.892 & 0.391 & 36.854 \\
0.411 & 17.493 & 0.411 & 36.854 \\
0.430 & 16.510 & 0.430 & 36.854 \\
0.450 & 21.722 & 0.450 & 36.854 \\
0.469 & 28.744 & 0.469 & 36.854 \\
0.489 & 29.298 & 0.489 & 36.854 \\
0.508 & 29.599 & 0.508 & 36.854 \\
0.528 & 29.885 & 0.528 & 36.854 \\
0.547 & 29.885 & 0.547 & 36.854 \\
0.567 & 29.885 & 0.567 & 36.854 \\
0.586 & 29.885 & 0.586 & 36.854 \\
\hline \hline
\end{tabular}

\section{CONCLUSION}

In this paper, the OMP and OMP-PKS algorithms use to reconstruct the watermarked compressive sensing measurements. The watermarked measurements consist of the original and watermarking images. The results compare the performance of the OMP and OMP-PKS algorithms. Experimental result explains that the OMP-PKS has a higher PSNR value in lower $M / N$ rates than the OMP.

In addition, the recovered Lena and extracted watermark images have a good quality in lower measurement rates by using the OMP-PKS than the OMP. Therefore, the OMP-PKS algorithm improves the performance, thereby 
requiring fewer samples than OMP to reconstruct the sparse signal. Also the OMP-PKS has a faster run time and more efficient than the OMP.

\section{REFERENCES}

[1] R. Baraniuk, "Compressive sensing," IEEE Signal Processing Mag, pp. 118-120, 2007.

[2] D. L. Donoho, "Compressed sensing," IEEE Trans. Inform. Theory, vol. 52, pp. 1289-1306, 2006.

[3] L. Jacques, "A short note on compressed sensing with partially known signal support," Signal Processing, vol. 90, no. 12, pp. 3308-3312, Dec. 2010

[4] M. Fornasier and H. Rauhut, "Compressive sensing," Part 2 of the Handbook of Mathematical Methods in Imaging, Springer, pp. 187-228, 2011.

[5] N. Vaswani and W. Lu, "Modified-CS: Modifying compressive sensing for problems with partially known support," in Proc. IEEE Intl. Symp. Info. Theory (ISIT), June 2009, pp. 488-492.

[6] J. A. Tropp and A. C. Gilbert, "Signal recovery from random measurements via orthogonal matching pursuit," IEEE Trans. Inf. Theory, vol. 53, pp. 4655-4666, Dec. 2007.

[7] R. E. Carrillo, L. F. Polania, and K. E. Barner, "Iterative algorithm for compressed sensing with partially know support," in Proc. IEEE ICASSP, Texas, U.S.A, 2010, pp. 3654-3657.

[8] D. Needell and R. Vershynin, "Signal recovery from incomplete and inaccurate measurements via regularized orthogonal matching pursuit," IEEE Journal of Selected Topics in Signal Processing, vol. 4, pp. 310-316, Apr. 2010.

[9] E. Muharemagić and B. Furht, "Survey of watermarking techniques and applications," in Multimedia Watermarking Techniques and Applications, B. Furht and D. Kirovski, Eds. Auerbach Publication, 2006, pp. 91-130.

[10] S. Stankovic, I. Djurovic, R. Herpers et al., "An approach to the optimal watermark detection," AEUE International Journal of Electronics and Communications, vol. 57, no. 5, 2003, pp. 355-357.

[11] G. Kutyniok, "Compressed sensing: Theory and applications," CoRR, vol. abs/1203.3815, 2012.
[12] S. Foucart and H. Rauhut, A Mathematical Introduction to Compressive Sensing, Birkhäuser, London, 2013.

[13] E. Candes and T. Tao, "Decoding by linear programming," IEEE Trans. Inf. Theory, vol. 51, no. 12, pp. 4203-4215, Dec. 2005.

[14] M. Yamac, C. Dikici, and B. Sankur, "Watermarking of compressive sensed measurements," in Proc. SPARS 2013,Lausanne, Jul. 2013.

[15] S. G. Mallat and Z. Zhang, "Matching pursuits with time-frequency dictionaries," IEEE Trans Signal Process, vol. 41, no. 12, pp. $3397-3415$

[16] P. Sermwuthisarn, S. Auethavekiat, D. Gansawat, and V. Patanavijit, "Robust reconstruction algorithm for compressed sensing in Gaussian noise environment using orthogonal matching pursuit with partially known support and random subsampling," EURASIP Journal on Advances in Signal Processing, vol. 34, 2012.

[17] P. Sermwuthisarn, D. Gansawat, V. Patanavijit, and S. Auethavekiat, "Impulsive noise rejection method for compressed measurement signal in compressed sensing," EURASIP Journal on Advances in Signal Processing, vol. 68, 2012.

[18] M. Yamac, C. Dikici, and B. Sankur, "Robust watermarking of compressive sensed measurements under impulsive and gaussian attacks," in Proc. 21st EUSIPCO, 2013, pp. 1-5.

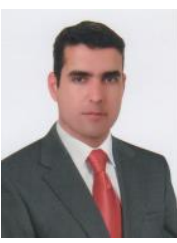

Ali A. H. Karah Bash was born in 1983 in Mosul, Iraq. He received the B.S. degree in electronic engineering from the University of Mosul in 2006. Now, he is a M.Sc. student in electrical and electronic engineering at University of Gaziantep, Turkey. His research interests include compressive sensing, watermarking techniques and statistical learning.

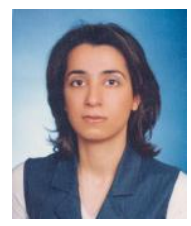

Sema Koç Kayhan was born in Gaziantep, Turkey in 1973. She received Ph.D. degree in electrical and electronic engineering from the University of Gaziantep, Turkey in 2005. Currently, she is an assistant professor of the Electrical and Electronic Engineering Department at Gaziantep University, Turkey, Her research interests include computer graphics, image and video processing. 